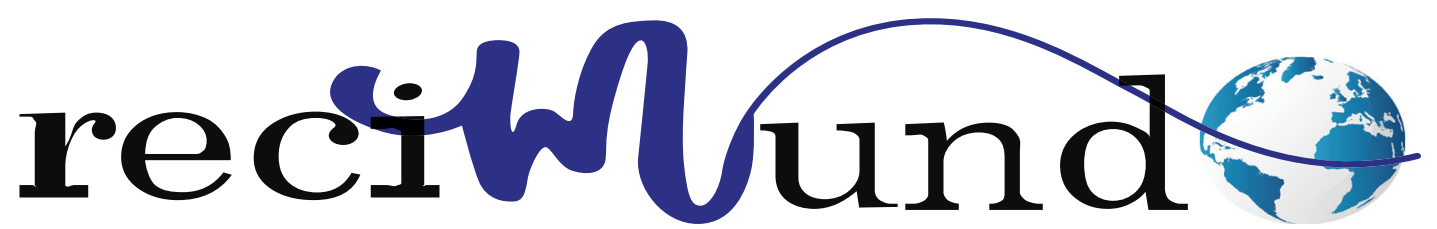

Revista Científica Mundo de la Investigación y el Conocimiento

DOI: 10.26820/recimundo/4.(4).octubre.2020.114-122

URL: http://recimundo.com/index.php/es/article/view/885

EDITORIAL: Saberes del Conocimiento

REVISTA: RECIMUNDO

ISSN: 2588-073X

TIPO DE INVESTIGACIÓN: Artículo de Revisión

CÓdIGO UNESCO: 3202 Epidemiología

PAGINAS: $114-122$

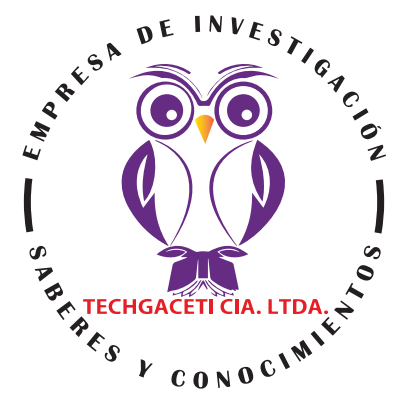

\title{
Consideraciones relativas a la discapacidad durante el brote de COVID-19
}

Disability considerations during the COVID-19 outbreak Considerações sobre deficiência durante o surto de COVID-19 Stalin Augusto Jurado Auria'; Sheyla Elizabeth Villacrés Caicedo²; Rosa María Bulgarín Sánchez ${ }^{3}$

RECIBIDO: 10/07/2020 ACEPTADO: 26/08/2020 PUBLICADO: 15/10/2020

1. Diploma Superior en Pedagogía Universitaria; Magister en Educación Superior; Licenciado en Terapia Física; Universidad Católica de Santiago de Guayaquil; Guayaquil, Ecuador; stalin.jurado@cu.ucsg.edu.ec iD https://orcid.org/0000-00021163-1030

2. Diplomado Superior en Diseño y Aplicación de Modelos Educativos, Diploma Superior en Diseño y Gestión Curricular, Magister en Diseño y Evaluación de Modelos Educativos, Licenciada en Terapia Física. Universidad Católica Santiago de Guayaquil; Guayaquil, Ecuador; sheyla.villacres@cu.ucsg.edu.ec (iD https://orcid.org/0000-0003-2306-8385

3. Magister en Gerencia en Servicios de la Salud; Doctora en Medicina y Cirugia; Universidad Católica Santiago de Guayaquil; Guayaquil, Ecuador; rosa.bulgarin@cu.ucsg.edu.ec iD https://orcid.org/0000-0001-7933-0253

CORRESPONDENCIA

Stalin Augusto Jurado Auria

stalin.jurado@cu.ucsg.edu.ec

Guayaquil, Ecuador

(C) RECIMUNDO; Editorial Saberes del Conocimiento, 2020 


\section{RESUMEN}

Dentro de la vida cotidiana existen personas invisibilizadas de las cuales se hace necesario aportar para evitar tal irresponsabilidad como seres humanos desde todos los espacios que se pueda trabajar. Las personas con todos los tipos de discapacidad deben poder gozar de todos los derechos humanos y libertades fundamentales. Hay más de mil millones de personas que viven con discapacidades alrededor del mundo. Nos hemos propuestos hacer una revisión bibliográfica que busca describir las declaraciones y manifestaciones de las principales organizaciones e investigadores relacionadas con los derechos de las personas con discapacidad durante la pandemia COVID-19. Se ha tomado como palabras claves discapacidad y COVID-19 para la búsqueda de publicaciones y documentos que puedan aportar al objetivo y poderlas plasmar de manera organizada y resumida en los resultados de la presente investigación. La pandemia, ya a estas alturas temporales de su desarrollo ha tenido un impacto emocional, el "detenimiento" del ritmo de vida ha logrado re-valorar el tiempo, la disposición y el uso de los recursos. Como punto reflexivo final, la vulnerabilidad generalizada respecto al virus nos ha hecho conscientes de la vulnerabilidad ajena y desde la concepción de las realidades individuales hemos logrado y estamos trabajando en una nueva valuación de nuestras oportunidades y sobretodo de nuestras capacidades de cuidar y ser cuidados.

Palabras clave: Personas con discapacidad, vulnerabilidad, COVID-19.

\section{ABSTRACT}

Within everyday life there are invisible people of which it is necessary to contribute to avoid such irresponsibility as human beings from all the spaces where it is possible to work. People with all types of disabilities must be able to enjoy all human rights and fundamental freedoms. There are more than one billion people living with disabilities around the world. We have proposed to make a bibliographic review that seeks to describe the statements and statements of the main organizations and researchers related to the rights of people with disabilities during the COVID-19 pandemic. Disability and COVID-19 have been taken as keywords for the search for publications and documents that can contribute to the objective and be able to capture them in an organized and summarized way in the results of this research. The pandemic, already at this time point in its development, has had an emotional impact, the "stopping" of the rhythm of life has managed to revalue the time, disposition and use of resources. As a final point of reflection, the generalized vulnerability to the virus has made us aware of the vulnerability of others and from the conception of individual realities we have achieved and are working on a new assessment of our opportunities and above all of our capacities to care and be cared for..

Keywords: People with disabilities, vulnerability, COVID-19.

\section{RESUMO}

No cotidiano existem pessoas invisíveis das quais é preciso contribuir para evitar a irresponsabilidade do ser humano de todos os espaços onde é possível trabalhar. Pessoas com todos os tipos de deficiência devem poder desfrutar de todos os direitos humanos e liberdades fundamentais. Existem mais de um bilhão de pessoas portadoras de deficiência em todo o mundo. Propomos fazer uma revisão bibliográfica que busca descrever as declarações e declarações das principais organizações e pesquisadores relacionadas aos direitos das pessoas com deficiência durante a pandemia COVID-19. Disability e COVID-19 têm sido tomados como palavras-chave para a busca de publicações e documentos que possam contribuir com o objetivo e ser capazes de captá-los de forma organizada e sintetizada nos resultados desta pesquisa. A pandemia, já nesta altura do seu desenvolvimento, teve um impacto emocional, a "paragem" do ritmo de vida conseguiu reavaliar o tempo, a disposição e o uso dos recursos. Como último ponto de reflexão, a vulnerabilidade generalizada ao vírus nos fez tomar consciência da vulnerabilidade alheia e da concepção das realidades individuais que alcançamos e estamos trabalhando em uma nova avaliação de nossas oportunidades e sobretudo de nossas capacidades de cuidado. e ser cuidado.

Palavras-chave: Pessoas com deficiência, vulnerabilidade, COVID-19. 


\section{Introducción}

Dentro de la vida cotidiana existen personas invisibilizadas de las cuales se hace necesario aportar para evitar tal irresponsabilidad como seres humanos desde todos los espacios que se pueda trabajar. Hoy se les identifica como personas con discapacidad y fue el diciembre de 2006 donde se aprueban sus derechos a través de La Convención sobre los derechos de las personas con discapacidad y su Protocolo Facultativo en la Sede de las Naciones Unidas. A partir de allí se adopta una amplia clasificación de las personas con discapacidad y se reafirma que todas las personas con todos los tipos de discapacidad deben poder gozar de todos los derechos humanos y libertades fundamentales. La División de Desarrollo social fue designada punto focal de la CEPAL para el seguimiento de la Convención sobre los Derechos de las Personas con Discapacidad y la coordinación de las iniciativas y actividades desarrolladas en el marco del programa de trabajo en el tema de discapacidad. (CEPAL-Naciones Unidas, 2020)

Hay más de mil millones de personas que viven con discapacidades alrededor del mundo. La OMS define discapacidad como "toda restricción o ausencia debida a una deficiencia, de la capacidad de realizar una actividad en la forma o dentro del margen considerado normal para el ser humano"; esta puede ser temporal o permanente, reversible o irreversible, cabe resaltar que la persona "no es" discapacitada, sino que "está" discapacitada. (Aquino Canchari, Quispe Arrieta, \& Huaman Castillon, 2020) Las epidemias han marcado cambios demográficos y sociales a lo largo de la historia. El nuevo coronavirus, Covid-19, ha cambiado nuestra percepción del mundo en pocas semanas, modificando muchas perspectivas. El aislamiento social ha supuesto un objetivo necesario no exento de consecuencias que han generado decisiones que, eran impensables. De forma im- prevista, los servicios sanitarios han tenido que adaptarse a la nueva realidad, tomándose decisiones en el beneficio de los pacientes, pero no exentas de consecuencias indirectas. (Guiu, Porta Etessam, López Valdes, García Morales, \& Guerrero Solá, 2020) El COVID-19, también conocido como coronavirus, es una enfermedad infecciosa causada por el virus SARS-CoV-2. Produce síntomas similares a los de la gripe, en casos graves conduce alrededor del 3\% de los infectados a la muerte. La transmisión se produce mediante pequeñas gotas que se emiten al hablar, estornudar, toser o espirar que, al ser despedidas por un portador (aunque éste no tenga síntomas de enfermedad), pasan directamente a otra persona mediante la inhalación, o quedan sobre los objetos y superficies desde donde pueden ser transportadas a través de las manos, y entrar en contacto con las membranas mucosas orales, nasales y oculares, siendo esta última la principal vía de propagación, los síntomas aparecen entre dos y catorce días, siendo la media de cinco, después de la exposición al virus. Ante la ausencia de tratamiento toman especial relevancia las medidas de prevención entre las que se encuentra evitar el contacto con otras personas. Pero más allá de la dramática pérdida de vidas, el virus ha hecho que nuestro mundo se pare. La actividad económica se ha reducido a los mínimos imprescindibles, las relaciones sociales han quedado bloqueadas, en standby hasta nueva orden y nos hemos visto forzados a un aislamiento no deseado. Todo ello, tiene consecuencias. Consecuencias sobre nuestra salud física y psíquica, consecuencias en el empleo, consecuencias sobre el futuro que hoy se dibuja incierto y desconcertante. (Silván \& Quiféz, 2020)

Considerando lo anterior nos hemos propuestos hacer una revisión bibliográfica que busca describir las declaraciones y manifestaciones de las principales organizaciones e investigadores relacionadas con los derechos de las personas con discapa- 
cidad durante la pandemia COVID-19.

\section{Metodología}

Se ha tomado como palabras claves discapacidad y COVID-19 para la búsqueda de publicaciones y documentos que puedan aportar al objetivo de conseguir las consideraciones relevantes de las personas discapacitadas y poderlas plasmar de manera organizada y resumida en los resultados de la presente investigación.

Utilizando herramientas como lectura rápida, resumen y análisis crítico se presentan a continuación los elementos encontrados de 3950 resultados que ha arrojado la búsqueda a través de Google Académico, de acuerdo a la relevancia se ha organizado la información descartando publicaciones en un idioma distinto al español.

\section{Resultados}

Venturiello, (2017); Díaz (2018); Pérez y Chabra (2019), sustentan la construcción sociohistórica de la discapacidad mediante la ruptura de las barreras que limitan el acceso al entorno, igualdad de oportunidades, el respeto a las diferencias y la participación social en el diseño de políticas públicas más inclusivas hacia esas personas. Desde la diversidad, Palacios y Romañach (2006) proponen una perspectiva que las personas con discapacidad conforman un grupo social con comportamientos, interacciones, comunicaciones, sistemas simbólicos, procesos de socialización, biografía individual y colectiva, constitutivos de su diversidad e inclusión educativa. Posturas teóricas recientes abordan a la discapacidad como construcción cultural conformada por estructuras de discriminación, marginación y exclusión que configuran la situación de estas personas (González, 2010). También se incluyen las barreras que excluyen, discriminan o estigmatizan a este grupo social en el acceso a programas educativos, las TIC y el aprendizaje virtual. La particula- ridad biológico conductual comprende las características individuales o colectivas de estas personas, sus prácticas, imaginarios culturales y los significados construidos en su modo de vida. (Peña Estrada, Vaillant Delis, Soler Nariño, Bring Pérez, \& Domínguez Ruiz, 2020)

La COVID-19 amenaza con exacerbar las disparidades de las personas, particularmente en países de bajos y medianos ingresos. Por ello, todo Estado se encuentra en la obligación de brindar garantías para las medidas de aislamiento preventivo obligatorio para este grupo de personas en cumplimiento de sus derechos. Es importante mencionar el impacto emocional que podría presentarse en la mayoría de los pacientes con discapacidades debido al aislamiento social y la interrupción de sus terapias diarias; estos cambios podrían propiciar comportamientos disruptivos para cada tipo de discapacidad, debido al incremento del estrés. (Aquino Canchari, Quispe Arrieta, \& Huaman Castillon, 2020)

La atención hacia las personas con discapacidad durante la pandemia del Covid-19 emanadas por las Organizaciones de atención, sobre todo las que defienden los derechos humanos se ha centrado en 4 elementos que observar.

1. Principio de igualdad y no discriminación

2. Derecho a la dignidad Inherente

3. Derecho a la accesibilidad

4. Derecho a la salud

Las medidas para proteger la salud física pueden tener efectos en el bienestar especialmente en países con bajos y medianos ingresos, sistemas de salud débiles y con limitados recursos. En estos países es necesario que las personas con discapacidad tengan acceso a una información incluyente en todas las lenguas y lenguaje de señas, en diferentes canales como la televisión, los teléfonos móviles y los periódicos. Utilizar

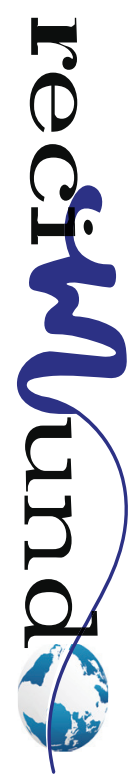


lenguajes simples y comprensibles para todos especialmente para las personas con discapacidad intelectual. (Lugo Agudelo, Patiño, \& Velez, 2020)

Las consecuencias primarias respecto al COVID- 19 en personas con discapacidad son aquellas relacionadas con el virus, como el mayor riesgo de contagio por falta de acceso a la información, la necesidad de continuar su asistencia a los centros de atención lo que les impide seguir las recomendaciones de distanciamiento físico, la mayor probabilidad de presentar formas graves de la enfermedad o la muerte y la exacerbación de enfermedades o condiciones mentales preexistentes. Las consecuencias secundarias están relacionadas con la disminución de acceso a los servicios y recursos en rehabilitación, las necesidades insatisfechas en la protección social, el desempleo o la imposibilidad de ejercer empleos informales y la vulnerabilidad a la discriminación, la negligencia, la violencia o el abuso. Tanto las personas con consecuencias de COVID-19, como todas las que tienen una discapacidad tienen derecho a ser atendidas en servicios de rehabilitación. "La rehabilitación es la estrategia de salud que permite que las personas con discapacidad alcancen y mantengan el funcionamiento óptimo. Es la estrategia de salud para el siglo XXI, parte de la cobertura universal en salud y debe estar disponible para todas las personas". En forma similar a todos los desastres, el tratamiento efectivo de la rehabilitación en la pandemia incluye el manejo de la condición, prevenir complicaciones, optimizar el funcionamiento, incluyendo las funciones cognitivas, neuropsicológicas así como las condiciones mentales; se debe favorecer el reintegro social aun en épocas de distanciamiento físico. Es muy importante generar conocimiento que permita construir un precedente de atención en casos como este, que se distancia por sus condiciones particulares de lo existente en rehabilitación en otras condiciones (Lugo Agudelo, Patiño, \& Velez, 2020)
El informe de la Organización Mundial de la Salud sobre discapacidad mostró que más de un billón de la población mundial (15\%) vive con alguna forma de discapacidad, de los cuales 2-4\% experimenta dificultades significativas en el funcionamiento. En tiempos de crisis, incluida las guerras y los desastres naturales, los niños con discapacidades enfrentan desafíos adicionales como resultado de sus limitaciones funcionales, pero lo más importante; las muchas barreras que la sociedad pone en su camino. La falta de respuesta humanitaria inclusiva, el abandono y la separación de los miembros de la familia son formas comunes de violación de los derechos humanos de estos niños. En vista de la pandemia de COVID-19 en curso, es importante crear conciencia sobre los derechos humanos individuales y colectivos para acceder a los servicios en forma equitativa y garantizar que TODAS las personas sean tratadas con dignidad y respeto. La prevención es la estrategia de promoción de la salud más poderosa. Principalmente, para lograr respuestas exitosas e inclusivas basadas en la comunidad, necesitamos asegurarnos de que las personas con discapacidades tengan roles y responsabilidades en el diseño e implementación de los planes. La Academia Latinoamericana de Desarrollo Infantil y Discapacidades (ALDID), invita a investigadores y clínicos, que trabajan en el campo de la discapacidad pediátrica, a abogar por estándares claros sobre respuestas humanitarias inclusivas en nuestras comunidades. Finalmente, construir sistemas integrales de atención de emergencias sanitarias, y garantizar que TODOS nos beneficiemos de un enfoque inclusivo basado en los derechos humanos tendrá un impacto positivo en el bienestar de las personas con discapacidad, sus familias y nuestras comunidades. (Schiariti, 2020)

La vulnerabilidad de las personas con discapacidad según el informe de la CEPAL se va disgregando por flancos. 


\section{En la salud:}

Existe una relación positiva entre edad y discapacidad, es razonable pensar que algunas de las personas mayores que padecen de COVID-19 o fallecieron por esta causa tenían alguna discapacidad. Además de su perfil etario, las personas con discapacidad tienen un mayor riesgo ante el COVID-19 debido a sus limitadas posibilidades de protegerse del contagio o de buscar diagnóstico y tratamiento por la falta de información sobre el virus en formatos accesibles y la falta de accesibilidad en los establecimientos de salud. (Cecchin \& Bárcena, 2020)

\section{En la economía:}

Según la CEPAL (2020), el sector más afectado por las medidas de distanciamiento social y cuarentena es el de los servicios, que depende de contactos interpersonales. Si se tiene en cuenta que las personas con discapacidad ya tenían un tenue vínculo con el mercado laboral y una participación importante en el sector de servicios y en el empleo informal, es previsible que esta población se vea especialmente afectada por el desfavorable escenario del mercado laboral durante la crisis y el período de recuperación. Aunque los datos concretos sobre esta realidad escasean, en una investigación en el Paraguay se estimó que el 40\% de las personas con discapacidad en ese país quedaron desempleadas tras el inicio de la cuarentena, con impactos significativos en los ingresos de sus hogares (ABC Color, 2020). (Cecchin \& Bárcena, 2020)

\section{En la educación:}

El ámbito escolar también presenta complejidades particulares para estudiantes con discapacidad y sus familias en el contexto de la pandemia. Según la CEPAL (2020), al 20 de marzo, 16 países (Argentina, Bolivia (Estado Plurinacional de), Chile, Colombia, el Ecuador, El Salvador, Guatemala, Hondu- ras, Jamaica, Panamá, el Paraguay, el Perú, Santa Lucía, Trinidad y Tabago, el Uruguay y Venezuela (República Bolivariana de)) habían suspendido las clases en todos los niveles educativos, y el Brasil había aplicado cierres localizados de centros educativos. La interrupción de las actividades en centros educativos tendrá efectos significativos en el aprendizaje de estudiantes con discapacidad y podría afectar también su estado nutricional, ya que muchos estudiantes de hogares vulnerables, incluidos niños, niñas y adolescentes con discapacidad, dependen de programas de alimentación escolar. Aunque se han hecho planes para promover el uso de dispositivos digitales en los sistemas educativos, aseguran Ullmann y otros (2018) quew existen brechas en el acceso a las computadoras y a Internet y limitaciones en las habilidades para su uso en los hogares con personas con discapacidad. El cierre de los establecimientos educativos y el traspaso de gran parte de la responsabilidad del acompañamiento y apoyo al aprendizaje de los docentes a los padres (principalmente las madres) también presenta un reto si estos no cuentan con las habilidades y técnicas didácticas para atender las necesidades de aprendizaje de sus hijos e hijas con discapacidad.

\section{En la calidad de vida:}

El confinamiento físico que se ha aplicado en muchos países de la región para frenar la transmisión del virus puede interrumpir el acceso a bienes y servicios esenciales para que las personas con discapacidad puedan mantener su bienestar. Esto es especialmente crítico en el caso de servicios de salud, rehabilitación y cuidados, pero también en el de medicamentos, pañales, dispositivos de asistencia y alimentos especiales, entre otros insumos. El confinamiento y las restricciones de desplazamiento también pueden agravar la situación de personas con ciertos tipos de discapacidad, por ejemplo, aquellas con trastorno del espectro autista (TEA), cuyas rutinas permiten

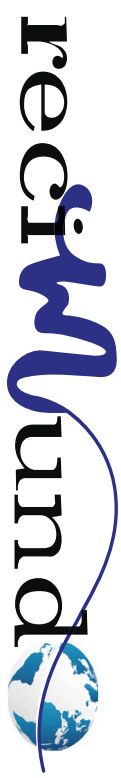


disminuir el estrés y la ansiedad. La interrupción de esas rutinas debido al confinamiento puede afectar de manera negativa el bienestar de esas personas y sus familias. El confinamiento puede aumentar la exposición a la violencia intrafamiliar en el caso de las mujeres con discapacidad, que son víctimas de este tipo de violencia en mayor medida que las mujeres sin discapacidad (Cecchin \& Bárcena, 2020).

Entre las recomendaciones emanadas de este informe se encuentran

1. Respetar los derechos fundamentales e inalienables de todas las personas en el contexto de la crisis y en el período de recuperación, independientemente de su situación de discapacidad, y atender en particular a subgrupos de la población que pueden estar en especial situación de desventaja, como mujeres, niños, niñas y adolescentes con discapacidad.

2. Garantizar la no discriminación en la provisión de bienes y servicios, y que todas las personas puedan acceder en igualdad de condiciones a programas y servicios de mitigación del gobierno.

3. Asegurar la accesibilidad a toda la información relacionada con la crisis.

4. Asegurar la accesibilidad en espacios donde se llevan a cabo las pruebas para diagnosticar y tratar el COVID-19.

5. Reforzar las prestaciones de protección social no contributivas para las personas con discapacidad y sus familias.

6. Velar por que las medidas tomadas para enfrentar la crisis y durante el período de recuperación, como las medidas sanitarias o las medidas sobre protección del empleo y continuidad educativa, incorporen la perspectiva de discapacidad.

7. Crear o ampliar espacios de participación y consulta con las organizaciones de personas con discapacidad.

8. Asegurar la continuidad del trabajo, la educación y la prestación de servicios de rehabilitación para las personas con discapacidad a través del uso de tecnologías de la información y las comunicaciones (TIC).

9. Proveer apoyo psicosocial a personas con discapacidad y sus familias y potenciar las redes de apoyo a personas con discapacidad.

10. Flexibilizar las restricciones a la circulación en la vía pública para personas con discapacidad y considerar la posibilidad de establecer horarios de atención diferenciados en los comercios para personas con discapacidad y otros grupos vulnerables al virus.

11. Mejorar la información estadística recopilada durante y después de la crisis, de manera que los países cuenten con información desagregada sobre los impactos diferenciados de la pandemia del COVID-19 sobre determinados grupos poblacionales, incluidos las personas con discapacidad. (Cecchin \& Bárcena, 2020)

\section{Conclusiones}

Algunos de estos esfuerzos en función de la atención y protección hacia las personas con discapacidad ya son un hecho, la limitante actual sería poder medir el impacto de dichos esfuerzos puesto que se consideran 2 elementos fundamentales.

El primero tiene que ver con el tiempo, la pandemia sigue en desarrollo, al menos para nuestra región, por lo tanto seguimos ajustándonos a la nueva realidad, sin embargo, ya se cuenta con algunas experiencias de cuidados que deben resaltarse como por ejemplo la flexibilización de las políticas de seguridad, contención y propagación del virus SARS-COV-2 para las per- 
sonas vulnerables, entre los que se resaltan la flexibilización del tránsito, la prioridad en la atención en centros de salud, las alternativas para suplir las necesidades entre otras.

La segunda y quizá la más profunda es que no existe estudio a datos disponibles que permitan generar políticas de mayor impacto hacia la población vulnerable, reconocido incluso por una organización como la CEPAL.

La puesta a disposición de información aun con el confinamiento ha permitido la toma de conciencia generando un nivel de corresponsabilidad y participación de las población vulnerables, es así como las comu-

\section{Bibliografía}

Aquino Canchari, C. R., Quispe Arrieta, R., \& Huaman Castillon, K. M. (2020). COVID-19 y su relación con poblaciones vulnerables. Revista Habanera de Ciencias Médicas, 19(1), e3341.

Cecchin, S., \& Bárcena, A. (03 de abril de 2020). Personas con discapacidad ante la enfermedad por coronavirus (COVID-19) en América Latina y el Caribe: situación y orientaciones. (N. CEPAL, Ed.) Obtenido de cepal.org: https://www.cepal. org/es/publicaciones/45491-personas-discapacidad-la-enfermedad-coronavirus-covid-19-america-latina-caribe

CEPAL-Naciones Unidas. (2020). Personas con Discapacidad. Recuperado el 03 de octubre de 2020, de cepal.org: https://www.cepal.org/es/temas/personas-discapacidad

Guiu, M., Porta Etessam, J., López Valdes, E., García Morales, A., \& Guerrero Solá, A. (2020). La gestión de la asistencia neurológica en tiempos de la Pandemia de Covid-19. Neurología, 35(4), 233-237.

Lugo Agudelo, L. R., Patiño, D., \& Velez, M. (2020). Derechos de las Personas con Discapacidad en la pandemia COVID-19. Síntesis rápida. Revista Colombiana de Medicina Física y Rehabilitación, https://www.researchgate.net/ profile/Marcela_Velez6/publication/344311803_ Derechos_de_las_Personas_con_Discapacidad_en_la_pandemia_COVID-19_Sintesis_rapida/links/5f663d3392851c14bc8849cd/ Derechos-de-las-Personas-con-Discapacidad-en-la-pandemia-COVID-19-Si. nidades mismas ha puesto en marcha sus propias medidas de atención y cuidados.

La pandemia, ya a estas alturas temporales de su desarrollo ha tenido un impacto emocional que ha volcado a la sociedad a un proceso de cambios desde el manejo del encierro, el "detenimiento" del ritmo de vida ha logrado re-valorar el tiempo, la disposición y el uso de los recursos. Como punto reflexivo final, la vulnerabilidad generalizada respecto al virus nos ha hecho conscientes de la vulnerabilidad ajena y desde la concepción de las realidades individuales hemos logrado y estamos trabajando en una nueva valuación de nuestras oportunidades y sobretodo de nuestras capacidades de cuidar y ser cuidados.

Peña Estrada, C., Vaillant Delis, M., Soler Nariño, O., Bring Pérez, Y., \& Domínguez Ruiz, Y. (2020). Personas con Discapacidad y Aprendizaje Virtual: Retos para las TIC en Tiempos de Covid-19. Revista Tecnológica-Educativa Docentes 2.0, 9(2), 204-211.

Schiariti, V. (2020). Los derechos humanos de los niños con discapacidad durante emergencias sanitarias: el desafío de COVID-19. DEVELOPMENTAL MEDICINE \& CHILD NEUROLOG, 62, E3-E4.

Silván, C., \& Quiféz, L. E. (2020). Efectos y consecuencias de la crisis de la COVID-19 entre las personas con discapacidad. Unión Europea. Fondo Social Europeo. Observatorio sobre Discapacidad y mercado de trabajo (ODISMET); Fundación ONCE. 

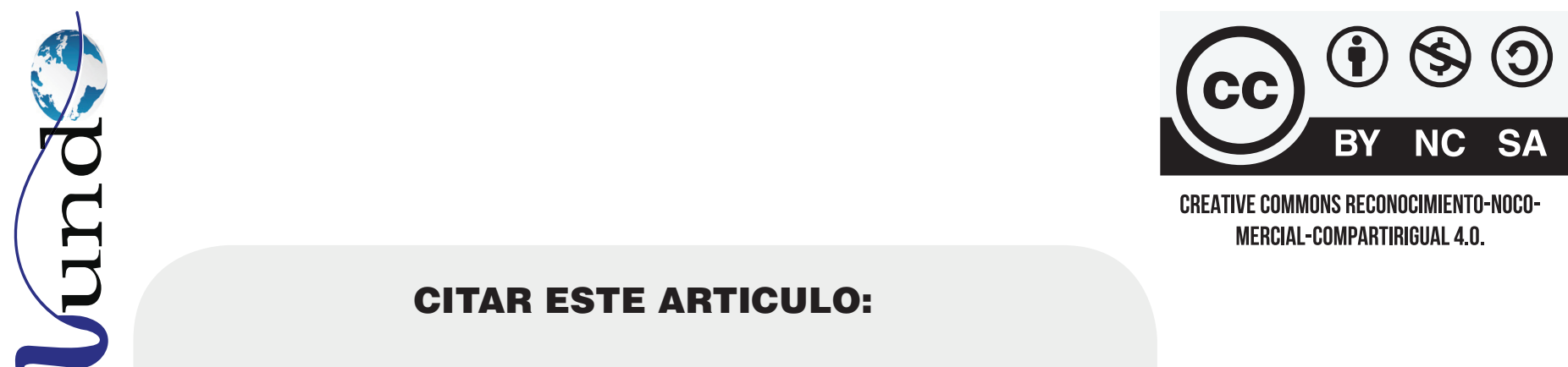

CREATIVE COMMONS RECONOCIMIENTO-NOCOMERCIAL-COMPARTIRIGUAL 4.0.

\section{CITAR ESTE ARTICULO:}

Jurado Auria, S. A., Villacrés Caicedo, S. E., \& Bulgarín Sánchez, R. M. (2020). Consideraciones relativas a la discapacidad durante el brote de COVID-19. RECIMUNDO, 4(4), 114-122. https://doi.org/10.26820/recimundo/4.(4).octubre.2020.114-122 